\title{
On binary pre-open sets and binary pre- $\omega$-open sets in binary topological spaces
}

\author{
Carlos Granados \\ Corporación Universitaria Latinoamericana, Colombia
}

Received: 14 June 2020, Accepted: 11 October 2020

Published online: 18 September 2021.

\begin{abstract}
As a generalization of binary open sets in binary topological, we used the notions of pre-open sets in topological spaces to introduce and study the notions of binary pre-open sets and binary pre- $\omega$-open sets in binary topological spaces. Furthermore. Besides, we develop some properties on binary pre- $\omega$-compact spaces and binary pre- $\omega$-connected spaces. Moreover, we define and discuss the concepts of binary pre-continuous functions and binary pre- $\omega$-continuous functions.
\end{abstract}

Keywords: Binary topological spaces, binary pre-open sets, binary pre- $\omega$-open sets, binary pre- $\omega$-compact spaces, binary pre- $\omega$ connected spaces, binary pre-continuous functions, binary pre- $\omega$-continuous functions.

\section{Introduction}

The notion of binary topological space was introduced by Nithyanantha and Thangavelu in 2011 [4], A binary topology from $X$ to $Y$ is a binary structure which satisfies the conditions of the Definition 1 that are analogous to the axioms of topology. This concept has been studied by many mathematicians in different fields of the general topology. In 2017, Mustafa [6] introduced the concept of binary generalized closed set in binary topological spaces in which he showed some properties in known open sets such that semi-open, pre-open, $b$-open, etc., in the next year, Chacko and Susha [1] used the notion of binary topological space to introduce the concept of binary linear topology in metric spaces. In 2019, Mehmood et.al. [5] used the notion of soft semi open sets to introduce the notion of soft binary topological spaces in which showed and proved some applications and characterizations in separation axioms. On the other hand, Hdeib [3] introduced the concept of $\omega$-closed set as generalized of closed sets. A point $x \in X$ is said to be a condensation point of $C$, if for each $V \in \tau$ with $x \in V$, the set $V \cap C$ is uncountable. $C$ is said to be $\omega$-closed [3], if it contains all its condensation points. The complement of a $\omega$-closed set is called $\omega$-open. The collection of all $\omega$-open sets of $(X, \tau)$ is denoted by $\tau \omega$ in which is finer than $\tau$. In this paper, as a generalization of binary open sets in binary topological spaces, we used the notions of pre-open sets [2] to introduce and study the notions of binary pre-open sets and binary pre- $\omega$-open sets, as well as, some of their properties and characterizations are shown. Besides, we introduce the notions of binary pre-continuous functions, binary pre- $\omega$-continuous functions, strongly binary pre- $\omega$-continuous functions and perfectly binary pre- $\omega$-continuous functions.

Throughout this paper, $P(X)$ and $P(Y)$ are the power sets of $X$ and $Y$, respectively. Now, we show some definitions which are useful for the developing of this paper.

Definition 1. [4]. Let $X, Y$ be any two empty sets and $A \subseteq X$ and $B \subseteq Y$. A binary topology from $X$ to $Y$ is a binary structure $M \subseteq P(X) \times P(Y)$ that satisfies the following conditions:

(1) $(0,0)$ and $(X, Y) \in M$. 
(2) $\left(A_{1} \cap A_{2}, B_{1} \cap B_{2}\right) \in M,\left(A_{1}, B_{1}\right) \in M$ and $\left(A_{2}, B_{2}\right) \in M$.

(3) If $\left\{\left(A_{\delta}, B_{\delta}\right): \delta \in \Delta\right\}$ is a family of members of $M$, then $\left(\underset{\delta \in \Delta}{\cup} A_{\delta}, \underset{\delta \in \Delta}{\cup} B_{\delta}\right) \in M$

Definition 2. [4]. If $M$ is a binary topology from $X$ to $Y$, then the triplet $(X, Y, M)$ is said to be a binary topological space and the members of $M$ are called binary open sets of $(X, Y, M)$. The elements of $X \times Y$ are said to be the binary points of the binary topological space $(X, Y, M)$.

Definition 3. [4]. Let $(X, Y, M)$ be a binary topological space and let $(x, y) \in(X, Y)$. The binary open set $(A, B)$ is said to be a binary neighbourhood of $(x, y)$ if $x \in A$ and $y \in B$.

Proposition 1. [4]. Let $(A, B) \subseteq(C, D) \subseteq(X, Y)$ and $(X, Y, M)$ be a binary topological space. Then, the following statements hold:

(1) $\operatorname{Int}(A, B) \subseteq(A, B)$.

(2) If $(A, B)$ is binary open, then $\operatorname{Int}(A, B)=(A, B)$.

(3) $\operatorname{Int}(A, B) \subseteq \operatorname{Int}(C, D)$.

(4) $\operatorname{Int}(\operatorname{Int}(A, B))=\operatorname{Int}(A, B)$.

(5) $(A, B) \subseteq C l(A, B)$.

(6) If $(A, B)$ is binary closed, then $C l(A, B)=(A, B)$.

(7) $C l(A, B) \subseteq C l(C, D)$.

(8) $\operatorname{Cl}(C l(A, B))=C l(A, B)$.

Definition 4. [4]. Let $f: Z \rightarrow X \times Y$ be a function. Let $A \subseteq X$ and $B \subseteq Y$. We define $f-1(A, B)=\{z \in Z: f(z)=(x, y) \in$ $(A, B)\}$.

Definition 5. [4]. Let $(X, Y, M)$ be a binary topological space and let $(Z, \tau)$ be a topological space. Now, let $f:(Z, \tau) \rightarrow$ $X \times Y$ be a function, then $f$ is said to be binary continuous if $f^{-1}(A, B)$ is open in $(Z, \tau)$ for every binary open set $(A, B)$ in $X \times Y$.

\section{Binary pre-open sets and binary pre-continuous functions}

In this section, we used the notion of binary open sets to introduce and study the notion of binary pre-open sets.

Definition 6. Let $(A, B)$ be a subset of a binary topological space $(X, Y, M)$. Then, $(A, B)$ is said to be binary pre-open if $(A, B) \subseteq \operatorname{Int}(C l(A, B))$. The complement of a binary pre-open set is called binary pre-closed.

Remark. The collection of all binary pre-open sets and binary pre-closed sets are denoted by $B P O(X, Y, M)$ and $B P C(X, Y$, $M)$, respectively.

Proposition 2. Every binary open set is binary pre-open.

Proof. Let $(A, B)$ be a binary open set in $(X, Y, M)$, then $(A, B) \subseteq \operatorname{Int}(C l(A, B))$, since $(A, B) \subseteq C l(A, B)=(C, D)$, indeed $(A, B) \subseteq \operatorname{Int}(C, D)$ and this proof that $(A, B)$ is a binary pre-open set.

The converse of the above Proposition need not be true as can be seen in the following example.

Example 1. Let $X=\{a, b, c\}, Y=\{1,2,3\}$ and $M=\{(0,0),(\{a\},\{1\}),(X, Y)\}$. Then, $(\{a, c\},\{1,3\})$ is a binary pre-open set, but it is not a binary open set.

Theorem 1. Let $(X, \tau)$ and $(Y, \sigma)$ be two any topological spaces. If $A$ and $B$ are pre-open in $X$ and $Y$, respectively. Then, $(A, B)$ is binary pre-open in $(X, Y, M)$. 
Proof. Let $A$ and $B$ be pre-open in $X$ and $Y$, respectively. Suppose that $(A, B)$ is not binary pre-open in $(X, Y, M)$, this implies that $(A, B) \operatorname{Int}(C l(A, B))$. Now, assume that $C l(A, B)=(C, D)$, then $(A, B) \operatorname{Int}(C, D)$ and so $\operatorname{Int}(C, D)=(0,0)$, therefore $\operatorname{Int}(C)=0$ and $\operatorname{Int}(D)=0$, where $A \subseteq C=C l(A)$ and $B \subseteq D=C l(B)$, hence $A$ and $B$ are not pre-open sets and this is a contradiction. Therefore, $(A, B)$ is a pre-open set.

Lemma 1. Let $A \subseteq X$ and $B \subseteq Y$. If $(A, B)$ is binary pre-open in $(X, Y, M)$, then $A$ is pre-open in $(X, \tau)$ and $B$ is pre-open in $(Y, \sigma)$.

Proof. The proof is followed by the Theorem 1.

Theorem 2.Let $(A, B)$ and $(C, D)$ be a binary open set in a binary topological space $(X, Y, M)$. Then, the following statements hold:

(1) $C l((A, B) \cup(C, D)) \supseteq C l(A, B) \cup C l(C, D)$.

(2) $C l((A, B) \cap(C, D)) \subseteq C l(A, B) \cap C l(C, D)$.

(3) $\operatorname{Int}((A, B) \cup(C, D)) \supseteq \operatorname{Int}(A, B) \cup \operatorname{Int}(C, D)$.

(4) $\operatorname{Int}(A, B) \cap \operatorname{Int}(C, D) \supseteq \operatorname{Int}((A, B) \cap(C, D))$.

Proof. (1) Let $(A, B) \subseteq(A, B) \cup(C, D)$ and $(C, D) \subseteq(A, B) \cup(C, D)$. Then, $C l(A, B) \subseteq C l((A, B) \cup(C, D))$ and $C l(C, D)$ $\subseteq C l((A, B) \cup(C, D))$ and hence $C l(A, B) \cup C l(C, D) \subseteq C l((A, B) \cup(C, D))$.

(2) Let $(A, B) \cap(C, D) \subseteq(A, B)$ and $(A, B) \cap(C, D) \subseteq(C, D)$. Then, $C l((A, B) \cap(C, D)) \subseteq C l(A, B)$ and $C l((A, B) \cap(C$, $D)) \subseteq C l(C, D)$ and hence $C l((A, B) \cap(C, D)) \subseteq C l(A, B) \cap C l(C, D)$.

(3) Let $(A, B) \subseteq(A, B) \cup(C, D)$ and $(C, D) \subseteq(A, B) \cup(C, D)$. Then, $\operatorname{Int}(A, B) \subseteq \operatorname{Int}((A, B) \cup(C, D))$ and $\operatorname{Int}(C, D) \subseteq$ $\operatorname{Int}((A, B) \cup(C, D))$ and hence $\operatorname{Int}(A, B) \cup \operatorname{Int}(C, D) \subseteq \operatorname{Int}((A, B) \cup(C, D))$.

(4) Let $(A, B) \cap(C, D) \subseteq(A, B)$ and $(A, B) \cap(C, D) \subseteq(C, D)$. Then, $\operatorname{Int}((A, B) \cap(C, D)) \subseteq \operatorname{Int}(A, B)$ and $\operatorname{Int}((A, B) \cap(C$, $D)) \subseteq \operatorname{Int}(C, D)$ and hence $\operatorname{Int}((A, B) \cap(C, D)) \subseteq \operatorname{Int}(A, B) \cap \operatorname{Int}(C, D)$.

The equality in part (1), (2), (3) and (4) in the above Theorem need not be true as can be seen in the following example.

Example 2. Let $X=\{1,2,3\}, Y=\{4,5\}$ and $M=\{(0,0),(X, Y),(\{1\},\{5\}),(\{2\}, Y),(\{1,2\}, Y)\}$.

Then, $C l(\{1\}, 0) \cup C l(\{2\}, 0)=(\{1,3\}, 0 /) \cup(\{2,3\},\{4\})=(X,\{4\})$, but $C l((\{1\}, 0) \cup(\{2\}, 0))=(X, Y)$.

Besides, $C l((\{1,2\},\{4\}) \cap(\{3\},\{5\}))=(\{3\}, 0)$, but $C l(\{1,2\},\{4\}) \cap C l(\{3\},\{5\})=(X, Y)$.

Now, $\operatorname{Int}(\{1\},\{4\}) \cup \operatorname{Int}(\{2\},\{5\})=(0,0)$, but $\operatorname{Int}((\{1\},\{4\}) \cup(\{2\},\{4\}))=(\{1,2\}, Y)$.

Besides, $\operatorname{Int}((\{1\},\{3\}) \cap(\{2\}, Y))=(0,0)$, but $\operatorname{Int}(\{1\},\{3\}) \cap \operatorname{Int}(\{2\}, Y)=(0,\{2\})$.

Theorem 3. Arbitrary union of binary pre-open sets is binary pre-open.

Proof. Let $\{(A, B) \delta: \delta \in \Delta\}$ be a collection of family of binary pre-open sets of $(X, Y, M)$, then $(A, B) \delta \subseteq \operatorname{Int}(C l((A, B \delta$ ))). Now, let $\left.\cup_{\delta \in \Delta}(A, B)_{\delta} \subseteq \cup_{\delta \in \Delta} \operatorname{Int}\left(C l\left((A, B)_{\delta}\right)\right)\right)$, by the Theorem 2 parts (1) and (3), we have that $\left.\cup_{\delta \in \Delta}(A, B)_{\delta} \subseteq \operatorname{Int}\left(C l\left(\cup_{\delta \in \Delta}(A, B)_{\delta}\right)\right)\right)$. Therefore, $\cup_{\delta \in \Delta}(A, B)_{\delta}$ is a binary pre-open set.

The following example shows that arbitrary intersection of binary pre-open sets need not be a binary pre-open set.

Example 3. Let $X=\{a, b, c\}, Y=\{1,2,3\}$ and $M=\{(0,0),(\{a\},\{1\}),(\{b\},\{2\}),(\{a, b\},\{1,2\}),(X, Y)\}$. Then, (\{a, $b\},\{1,2\})$ and $(\{a, 0 /\},\{1,2\})$ are binary pre-open sets, but $(\{a, b\},\{1,2\}) \cap(\{a, 0\},\{1,2\})=(\{a, 0\},\{1,2\})$ is not a binary pre-open set.

Definition 7. Let $(A, B)$ be a subset of a binary topological space $(X, Y, M)$. Then, $(A, B)$ is said to be binary pre-closed if $(A, B) \supseteq \operatorname{Int}(C l(A, B))$.

Theorem 4. Arbitrary intersection of binary pre-closed sets need not be a binary pre-closed set. 
Proof. Let $\left\{(A, B)_{\delta}: \delta \in \Delta\right\}$ be a collection of family of binary pre-closed sets of $(X, Y, M)$, then $(A, B)_{\delta} \supseteq \operatorname{Int}\left(C l\left(\left(A, B_{\delta}\right)\right)\right)$. Now, let $\left.\cap_{\delta \in \Delta}(A, B)_{\delta} \supseteq \cap_{\delta \in \Delta} \operatorname{Int}\left(C l\left((A, B)_{\delta}\right)\right)\right)$. by the Theorem 2 parts (2) and (4), we have that $\left.\left.\cap_{\delta \in \Delta}(A, B)_{\delta} \supseteq \cap_{\delta \in \Delta} \operatorname{Int}\left(C l\left((A, B)_{\delta}\right)\right)\right) \supseteq \operatorname{Int}\left(C l\left(\cap_{\delta \in \Delta}(A, B)_{\delta}\right)\right)\right)$. Therefore, $\cap_{\delta \in \Delta}(A, B)_{\delta}$ is a binary

The following example shows that arbitrary union of binary pre-closed sets need not be a binary pre-closed set.

Example 4. By the example 3, $(\{a\},\{1\})$ and $(\{b\},\{2\})$ are binary pre-closed sets, but $(\{a\},\{1\}) \cup(\{b\},\{2\})=(\{a$, $b\},\{1,2\})$ is not a binary pre-closed set.

Definition 8. Let $(X, Y, M)$ be a binary topological space and let $(Z, \tau)$ be a topological space. Now, let $f:(Z, \tau) \rightarrow X \times Y$ be a function, then $f$ is said to be binary pre-continuous if $f^{-1}(A, B)$ is open in $(Z, \tau)$ for every binary pre-open set $(A$, B) in $X \times Y$.

Theorem 5. Every binary continuous function is binary pre-continuous.

Proof. It follows form the fact that every binary open is binary pre-open.

The converse of the above Theorem need not be true as can be seen in the following example.

Example 5.Let $X=\{a, b, c\}, Y=\{0,1\}, M=\{(0,0),(\{a\},\{1\}),(\{b\},\{0,1\}),(X, Y)\}, Z=\{q, w, e\}$ and $\tau=\{0, Z$, $\{q\},\{w\},\{q, w\}\}$. Define the function $f:(Z, \tau) \rightarrow X \times Y$ by $f(q)=(a, 1), f(w)=(b, 0)$ and $f(e)=(c, 1)$. Then, $f$ is a binary pre-continuous function, but it is not a binary continuous function, because $f^{-1}(\{a, c\},\{2\})=\{q, w\}$ is not an open set.

\section{Binary pre- $\omega$-open sets and binary pre- $\omega$-continuous functions}

In this section, we used the notion of binary open sets and binary pre-open sets to introduce and study the notion of binary pre- $\omega$-open sets.

Definition 9. Let $(X, Y, M)$ be a binary topological space and $(A, B) \subseteq X \times Y$. Then $(A, B)$ is said to be binary pre- $\omega$ -open if for each $(x, y) \in(A, B)$ there exits a binary pre-open set $(V, U) x$ containing $(x, y)$ such that $(V, U) x-(A, B)$ is a countable set. The complement of a binary pre- $\omega$-open set is called binary pre- $\omega$-closed set.

Remark. The collection of all binary pre- $\omega$-open sets and binary pre- $\omega$-closed sets are denoted by $B P \omega O(X, Y, M)$ and $B P \omega C(X, Y, M)$.

Lemma 2.Every binary pre-open set is binary pre- $\omega$-open.

Proof. The proof is followed by the Definition 9.

The converse of the above Lemma need not be true as can be seen in the following example.

Example 6. Let $X=\{a, b, c, d\}, Y=\{q, w, e, r\}$ and $M=\{(0,0),(\{a\},\{w\}),(\{c\},\{r\}),(X, Y),(\{a, c\},\{w, r\})\}$. Then, $(\{d\}, 0)$ is a binary pre- $\omega$-open set, but it is not a binary pre-open set.

Lemma 3. Let $(X, Y, M)$ and $\left(X_{1}, Y_{1}, M\right)$ be two binary topological spaces such that $(A, B) \subseteq\left(X_{1}, Y_{1}\right)$ and $X_{1} \subseteq X$ and $Y_{1}$ $\subseteq Y$. If $(A, B)$ is a binary pre- $\omega$-open set of $(X, Y, M)$, then $(A, B)$ is a binary pre- $\omega$-open set of $\left(X_{1}, Y_{1}, M\right)$.

Proof. Let $(A, B)$ be a binary pre- $\omega$-open set of $(X, Y, M)$. Then, for every $(x, y) \in(A, B)$, there exits a pre- $\omega$-open set $(U, V)$ of $(X, Y, M)$ containing $(x, y)$ such that $(U, V)-(A, B)$ is a countable. In consequence, we have that $(U, V)$ is a pre- $\omega$-open set of $\left(X_{1}, Y_{1}, M\right)$ containing $(x, y)$. This proofs that $(A, B)$ is a pre- $\omega$-open set of $\left(X_{1}, Y_{1}, M\right)$. 
Theorem 6. Let $(X, Y, M)$ be a binary topological space and $(A, B) \subseteq(X, Y)$. Then $(A, B)$ is said to be binary pre- $\omega$ -open if and only if for every $(x, y) \in(A, B)$, there exits a binary pre-open set $(U, V) x$ containing $(x, y)$ and a countable subset $(A, B)$ such that $(U, V) x-(C, D) \subseteq(A, B)$.

Proof. Necessary: Let $(A, B)$ be a binary pre- $\omega$-open set and $(x, y) \in(A, B)$, then there exits a binary pre-open set $(U, V$ )$x$ containing $(x, y)$ such that $(U, V) x-(A, B)$ is countable. Now, let $(C, D)=(U, V) x-(A, B)=(U, V) x \cap((X-A), X-$ $B)$ ). Then, $(U, V) x-(C, D) \subseteq(A, B)$.

Sufficiency: Let $(x, y) \in(A, B)$. Then, there exits a binary pre- $\omega$-open sets $(U, V) x$ containing $(x, y)$ and a countable set $(C, D)$ such that $(U, V) x-(C, D) \subseteq(A, B)$. Therefore, $(U, V) x-(A, B) \subseteq(C, D)$ and $(U, V) x-(A, B)$ is countable.

Definition 10. Let $\{\psi \delta: \delta \in \Delta\}$ be a collection of binary pre-open sets in a binary topological space $(X, Y, M)$ is said to be a binary pre-open cover of a subset $(A, B)$ of $(X, Y)$ if $(A, B)(A, B) \subseteq \bigcup_{\delta \in \Delta} \psi_{\delta}$

Definition 11. Let $(X, Y, M)$ be a binary topological space. Then, $(X, Y)$ is said to be binary-pre-Lindeloff, if every binary pre-open cover of $(X, Y)$ has a countable sub-cover.

Theorem 7. Let $(X, Y, M)$ be a binary topological space. Then, the following statements are equivalent:

(1) $(X, Y)$ is binary-pre-Lindeloff.

(2) Every countable cover of $(X, Y)$ by binary pre-open sets has a countable sub-cover.

Proof.(2) $\Rightarrow$ (1): Since every binary pre-open set is binary pre- $\omega$-open set, the proof follows.

$(1) \Rightarrow(2)$ : Let $\left\{\psi_{\delta}: \delta \in \Delta\right\}$ be a cover of $(X, Y)$ by binary pre- $\omega$-open sets of $(X, Y)$. Now, for each $(x, y) \in(X, Y)$ there exits a $\delta(x, y) \in \Delta$ such that $(x, y) \in \psi_{\delta_{x, y}}$. Since $(U, V) \delta(x, y)$ is a binary pre- $\omega$-open. Then, there exits a binary pre-open set $(N, M)_{\delta_{x, y}}$ such that $(x, y) \in(N, M)_{\delta_{x, y}}$ and $(N, M)_{\delta_{x, y}}-(U, V)_{\delta_{x, y}}$ is countable. Then, the family $\left\{(N, M)_{\delta}: \delta \in \Delta\right\}$ is a binary cover of $(X, Y)$ and $(X, Y)$ is binary-pre-Lindeloff. Therefore, there exits a countable sub-cover $\delta_{(x, y)_{i}}$ with $i \in I$ such that $(X, Y)=\cup_{i \in I}(N, M)_{\delta_{(x, y)_{i}}}$, since

$$
(X, Y)=\cup_{i \in I}\left[\left((N, M)_{\delta_{(x, y)_{i}}}-(U, V)_{\delta_{(x, y)_{i}}}\right) \cup(U, V)_{\delta_{(x, y)_{i}}}\right]=\cup_{i \in I}\left[\left((N, M)_{\delta_{(x, y)_{i}}}-(U, V)_{\delta_{(x, y)_{i}}}\right) \cup \bigcup_{i \in I}(U, V)_{\delta_{(x, y)_{i}}}\right]
$$

Since $(N, M)_{\delta_{(x, y)_{i}}}-(U, V)_{\delta_{(x, y)_{i}}}$ is a countable set, for each $\delta\left((x, y)_{i}\right)$, there exists a countable subset $\Delta_{\delta\left((x, y)_{i}\right)}$ of $\Delta$ such that $(N, M) \delta_{(x, y)_{i}}-(U, V)_{\delta_{(x, y)_{i}}} \subseteq \underset{\Delta_{\delta\left((x, y)_{i}\right)}}{\bigcup}(U, V)_{\delta}$ and therefore $(X, Y) \subseteq \bigcup_{i \in I}\left(\underset{\delta \in \Delta_{\delta\left((x, y)_{i}\right)}}{\bigcup}(U, V)_{\delta}\right) \bigcup\left(\bigcup_{i \in I}(U, V)_{\delta_{(x, y)_{i}}}\right)$.

Theorem 8. Let $(X, Y, M)$ be a binary topological space and $(C, D) \subseteq(X, Y)$. If $(A, B)$ is a binary pre- $\omega$-closed set. Then, $(C, D) \subseteq(J, K) \cup(A, B)$, for some binary pre- $\omega$-closed set $(J, K)$ and a countable set $(A, B)$.

Proof. If $(C, D)$ is a binary pre- $\omega$-closed set. Then, $(X-C, X-D)$ is a binary pre- $\omega$-open set and hence by Theorem 6 , for every $(x, y) \in(X-C, X-D)$, there exits a binary pre- $\omega$-open set $(U, V)$ containing $(x, y)$ and a countable set $(A, B)$ such that $(U-A, V-B) \subseteq(X-C, X-D)$. Thus, $(C, D) \subseteq((X-(U-A),(X-(V-B)))=X-((U, V) \cap((X-A),(X$ $-B)))=((X-U),(X-V)) \cup(A, B)$, let $(J, K)=(X-U, X-V)$. Then, $(J, K)$ is a binary pre- $\omega$-closed set such that $(C, D) \subseteq(J, K) \cup(A, B)$.

Theorem 9. The union of any family of binary pre- $\omega$-open sets is binary pre- $\omega$-open set.

Proof. Let $\left\{(A, B)_{\delta}: \delta \in \Delta\right\}$ is a collection of binary pre- $\omega$-open subsets of $(X, Y)$. Then, for every $(x, y) \in \bigcup_{\delta \in \Delta}(A, B)_{\delta}$, $(x, y) \in(A, B)_{\delta}$, for some $\delta \in \Delta$. Hence, there exits a binary pre- $\omega$-open subset $(U, V)$ containing $(x, y)$, such that $U-$ $A, V-B)_{\delta}$ is countable. Now, as $\left(\left(U-\left(\bigcup_{\delta \in \Delta} A_{\delta}\right),\left(V-\left(\bigcup_{\delta \in \Delta} B_{\delta}\right)\right) \subseteq(U-A, V-B)_{\delta}\right.\right.$, and thus $\left(\left(U-\left(\bigcup_{\delta \in \Delta} A_{\delta}\right),\left(V-\left(\bigcup_{\delta \in \Delta} B_{\delta}\right)\right)\right.\right.$ is countable. Therefore, $\bigcup_{\delta \in \Delta}(A, B)_{\delta}$ is a binary pre- $\omega$-open set. 
Definition 12. The union of all binary pre- $\omega$-open sets contained in $(A, B) \subseteq(X, Y)$ is called binary pre- $\omega$-interior of $(A, B)$ and is denoted by Intp $\omega(A, B)$.

Definition 13. The intersection of all binary pre- $\omega$-closed sets of $(X, Y)$ containing $(A, B)$ is called binary pre- $\omega$-closure of $(A, B)$ and is denoted by $C l p \omega(A, B)$. The Intp $\omega(A, B)$ is a binary pre- $\omega$-open set and the Clp $\omega(A, B)$ is a binary pre- $\omega$-closed set.

Theorem 10. Let $(X, Y, M)$ be a binary topological space and $(A, B),(C, D) \subseteq(X, Y)$. Then, the following statements hold:

(1) $\operatorname{Intp} \omega(\operatorname{Intp} \omega(A, B))=\operatorname{Intp} \omega(A, B)$.

(2) if $(A, B) \subset(C, D)$, then Intp $\omega(A, B) \subset \operatorname{Intp} \omega(C, D)$.

(3) $\operatorname{Intp} \omega((A, B) \cap(C, D)) \subset \operatorname{Intp} \omega(A, B) \cap \operatorname{Intp} \omega(C, D)$.

(4) $\operatorname{Intp} \omega(A, B) \cup \operatorname{Intp} \omega(C, D) \subset \operatorname{Intp} \omega((A, B) \cup(C, D))$.

(5) Intp $\omega(A, B)$ is the largest binary pre- $\omega$-open subset of $(X, Y)$. contained in $(A, B)$.

(6) $(A, B)$ is binary pre- $\omega$-open if and only if $(A, B)=\operatorname{Intp} \omega(A, B)$.

(7) $\operatorname{Clp} \omega(\operatorname{Clp} \omega(A, B))=\operatorname{Clp} \omega(A, B)$.

(8) If $(A, B) \subset(C, D)$, then $C l p \omega(A, B) \subset C l p \omega(C, D)$.

(9) $C l p \omega(A, B) \cup C \operatorname{lp} \omega(C, D) \subset C \operatorname{Clp} \omega((A, B) \cup(C, D))$.

(10) $C l p \omega((A, B) \cap(C, D)) \subset C l p \omega((A, B)) \cap C l p \omega(C, D)$.

Proof. (1), (2), (6), (7) and (8) are follow directly from the Definition 9. (3), (4) and (5) are follow from part (2) of this Theorem. (9) and (10) are follow by applying part (8) of this Theorem.

Theorem 11. Let $(X, Y, M)$ be a binary topological space and $(A, B) \subset(X, Y)$. Then, the following statements hold:

(1) $\operatorname{Clp} \omega((X-A), Y-B))=(X, Y)-C l p \omega(A, B)$.

(2) $\operatorname{Intp} \omega((X-A),(Y-B))=(X, Y)-\operatorname{Intp} \omega(A, B)$.

Proof. We will prove (1) and (2), then:

(1) Let $(x, y) \in(X, Y)-C l p \omega(A, B)$. Then, there exits $(U, V) \in B P \omega O(X, Y, M)$ such that $(U, V) \cap(A, B)=0 /$ and hence it has $(x, y) \in \operatorname{Intp} \omega(A, B)$. This shows that $(X, Y)-C l p \omega(A, B) \subset \operatorname{Intp} \omega((X-A),(X-B))$. Now, take $(x$, $y) \in \operatorname{Intp} \omega((X-A),(X-B))$. Since Intp $\omega((X-A),(X-B)) \cap(A, B)=0 /$, it gets that $(x, y) \in / C l p \omega(A, B)$. In consequence, $C \operatorname{lp} \omega((X-A),(X-B))=(X, Y)-\operatorname{Intp} \omega(A, B)$.

(2) Let $(x, y) \in(X, Y)-\operatorname{Intp} \omega((X-A),(X-B))$. Since Intp $\omega((X-A),(X-B)) \cap(A, B)=0 /$, we have that $(x, y)$ $\in / C l p \omega(A, B)$ and this implies that $(x, y) \in(X, Y)-\operatorname{Clp} \omega(A, B)$. Now, take $(x, y) \in(X, Y)-C l p \omega(A, B)$. Then, there exist $(U, V) \in B P \omega O(X, Y, M)$ such that $(U, V) \cap(A, B)=0$. Therefore, Intp $\omega((X-A),(X-B))=(X, Y)-$ $\operatorname{Clp\omega }(A, B)$.

Definition 14. Let $(X, Y, M)$ be a binary topological space and $(A, B) \subseteq(X, Y)$. Then $(A, B)$ is said to be binary pre- $\omega$ neighbourhood of a point $(x, y) \in(X, Y)$ if there exists a binary pre- $\omega$-open set $(J, K)$ such that $(x, y) \in(J, K) \subset(A, B)$.

Theorem 12. Let $(X, Y, M))$ be a binary topological space and $(A, B) \subseteq(X, Y)$. Then, $(A, B)$ is binary pre- $\omega$-open set if and only if it is a binary pre- $\omega$-neighbourhood of each of its points.

Proof. Necessary: Let $(A, B)$ be a binary pre- $\omega$-open set of $(X, Y)$. Then by the Definition $14(A, B)$ is a binary pre- $\omega$ neighbourhood of each of its points.

Sufficiency: If $(A, B)$ is a binary pre- $\omega$-neighbourhood of each of its points. Then, for each $(x, y) \in(A, B)$, there exits $(C$, $D) x \in B P \omega O(X, Y, M)$ such that $(C, D) x \subset(A, B)$. In consequence, $(A, B)=\{(C, D) x:(x, y) \in(A, B)\}$. Since, each $(C$, $D) x$ is a binary pre- $\omega$-open and arbitrary union of binary pre- $\omega$-open sets is a binary pre- $\omega$-open set. therefore, $(A, B)$ is a binary pre- $\omega$-open set of $(X, Y)$. 
Definition 15. Let $(X, Y, M)$ be a binary topological space, then $(X, Y, M)$ is said to be binary pre- $\omega$-compact if every cover of $(X, Y)$ by binary pre- $\omega$-open sets has a finite subcover.

Theorem 13. Let $(X, Y, M)$ be a binary topological space, then $(X, Y, M)$ is binary pre- $\omega$-compact if and only if for every collection $\left\{(A, B)_{\alpha}: \alpha \in \Delta\right\}$ of binary pre- $\omega$-closed sets in $(X, Y, M)$ satisfying $\bigcap\left\{(A, B)_{\alpha}: \alpha \in \Delta\right\}=0$, there is a finite subcollection $(A, B)_{\alpha_{1}},(A, B)_{\alpha_{2}}, \ldots,(A, B)_{\alpha_{n}}$ with $\bigcap\left\{(A, B)_{\alpha_{k}}: k=1, \ldots, n\right\}=0$.

Proof. Let $\left\{(A, B)_{\alpha}: \alpha \in \Delta\right\}$ be a collection of binary pre- $\omega$-closed sets such that $\bigcap\left\{(A, B)_{\alpha}: \alpha \in \Delta\right\}=0$, then $\{(X-$ $\left.A, Y-B)_{\alpha}: \alpha \in \Delta\right\}$ is a collection of binary sets such that

$$
(X, Y)=(X, Y)-0=(X, Y)-\cap\left\{(A, B)_{\alpha}: \alpha \varepsilon \Delta\right\}=\cup\left\{(X-A, Y-B)_{\alpha}: \alpha \varepsilon \Delta\right\},
$$

That is $\left\{(X-A, Y-B)_{\alpha}: \alpha \varepsilon \Delta\right\}$ is a cover of $(X, Y)$ by binary pre- $\omega$-open sets. Since $(X, Y, M)$ is binary pre- $\omega$-compact, there exists a finite subcollection $(X-A, Y-B)_{\alpha_{1}},(X-A, Y-B)_{\alpha_{2}}, \ldots,(X-A, Y-B)_{\alpha_{n}}$ such that

$$
(X, Y)=\cup\left\{(X-A, Y-B) \alpha_{k}: k=1, \ldots, n\right\}=(X, Y)-\cap\left\{(A, B) \alpha_{k}: k=1, \ldots, n\right\} .
$$

This shows that $\cap\left\{(A, B) \alpha_{k}: k=1, \ldots, n\right\}=0$. Conversely, suppose that $\left\{(U, V)_{\alpha}: \alpha \in \Delta\right\}$ is a cover of $(X, Y)$ by binary pre- $\omega$-open sets, then $\left\{(X-U, Y-V)_{\alpha}: \alpha \in \Delta\right\}$ is a collection of binary pre- $\omega$-closed sets such that $\cap\left\{(X-U, Y-V)_{\alpha}\right.$ : $\alpha \in \Delta=(X, Y)-\cup\left\{(U, V)_{\alpha}: \alpha \in \Delta\right\}=(X, Y)-(X, Y)=0$. By hypothesis, there exists a fine subcollection

$$
(X-U, Y-V)_{\alpha_{1}},(X-U, Y-V)_{\alpha_{2}}, \ldots,(X-U, Y-Y)_{\alpha_{n}}
$$

such that

$$
\cap\left\{(X-U, Y-V)_{\alpha_{k}}: k=1, \ldots, n\right\}=0 .
$$

Follows

$$
\begin{aligned}
(X, Y) & =(X, Y)-0=(X, Y)-\cap\left\{(X-U, Y-V)_{\alpha_{k}}: k=1, \ldots, n\right\} \\
& =(X, Y)-\left((X, Y)-\cup\left\{(U, V)_{\alpha_{k}}: k=1, \ldots n\right\}\right)=\cup\left\{(U, V)_{\alpha_{k}}: k=1, \ldots, n\right\} .
\end{aligned}
$$

This shows that $(X, Y, M)$ is binary pre- $\omega$-compact.

Definition 16. Let $(X, Y, M)$ be a binary topological space, then $(X, Y, M)$ is said to be binary pre-connected if $(X, Y)$ cannot be written as a disjoint union of two non-empty binary pre-open sets.

Definition 17. Let $(X, Y, M)$ be a binary topological space, then $(X, Y, M)$ is said to be binary pre- $\omega$-connected if $(X, Y)$ cannot be written as a disjoint union of two non-empty binary pre- $\omega$-open sets.

Theorem 14. Let $(X, Y, M)$ be a binary topological space. If $(X, Y, M)$ is binary pre- $\omega$-connected, then $(X, Y, M)$ is binary pre-connected.

Proof. Let $(X, Y, M)$ be binary pre- $\omega$-connected. Now, Suppose that $(X, Y, M)$ is not binary pre-connected, then there exist non-empty binary pre-open sets $(A, B)$ and $(C, D)$ such that $(A, B) \cap(C, D)=0$ and $(A, B) \cup(C, D)=(X, Y)$. Then, by the Proposition 2, we have that $(A, B)$ and $(C, D)$ are binary pre- $\omega$-open sets and so, $(X, Y, M)$ is not binary pre- $\omega$-connected and this is a contradiction, therefore $(X, Y, M)$ is binary pre-connected.

Theorem 15. For a binary topological space $(X, Y, M)$, the following statements are equivalent:

(1) $(X, Y, M)$ is binary pre- $\omega$-connected.

(2) $(O, O)$ and $(X, Y)$ are the only subsets of $(X, Y)$ which are both binary pre- $\omega$-open and binary pre- $\omega$-closed. 
Proof. $(1) \Rightarrow(2)$ Let $(V, U)$ be a subset of $(X, Y)$ which is both binary pre- $\omega$-open and binary pre- $\omega$-closed, then $(X-$ $V, X-U)$ is both binary pre- $\omega$-open and binary pre- $\omega$-closed, so $(X, Y)=(V, U) \cup(X-V, X-U)$. Since $(X, Y, M)$ is binary pre- $\omega$-connected, then one of those sets is $(0,0)$. Therefore, $(V, U)=(0,0)$ or $(V, U)=(X, Y)$.

$(2) \Rightarrow(1)$ Suppose that $(X, Y, M)$ is not binary pre- $\omega$-connected and let $(X, Y)=(U, N) \cup(V, M)$, where $(U, N)$ and $(V$, $M)$ are disjoint non-empty binary pre- $\omega$-open sets in $(X, Y, M)$, then $(U, N)=(X, Y)-(V, M)$ is both binary pre- $\omega$-open and binary pre- $\omega$-closed. By hypothesis, $(U, N)=(0,0)$ or $(U, N)=(X, Y)$, which is a contradiction. Therefore, $(X, Y$, $M)$ ) is binary pre- $\omega$-connected.

Now, in this part, we define the concepts of binary pre- $\omega$-continuous functions. Moreover, we prove some of their properties.

Definition 18. Let $(X, Y, M)$ be a binary topological space and let $(Z, \tau)$ be a topological space. Now, let $f:(Z, \tau) \rightarrow X$ $\times Y$ be a function, then $f$ is said to be binary pre- $\omega$-continuous if $f^{-1}(A, B)$ is open in $(Z, \tau)$ for every binary pre- $\omega$-open $\operatorname{set}(A, B)$ in $X \times Y$.

Theorem 16. Every binary pre-continuous function is binary pre- $\omega$-continuous. Proof.It follows form the fact that every binary pre-open is binary pre- $\omega$-open.

The converse of the above Theorem need not be true as can be seen in the following example.

Example 7. Let $X=\{a, b, c\}, Y=\{0,1\}, M=\{(0,0),(\{a\},\{1\}),(\{b\},\{0,1\}),(X, Y)\}, Z=\{q, w, e\}$ and $\tau=\{0, Z$, $\{q\},\{w\},\{q, w\}\}$. Define the function $f:(Z, \tau) \rightarrow X \times Y$ by $f(q)=(a, 1), f(w)=(b, 0)$ and $f(e)=(c, 1)$. Then, $f$ is a binary pre- $\omega$-continuous function, but it is not a binary pre-continuous function, because $f^{-1}(\{a, c\},\{1\})=\{q, e\}$ is not a semi-open set.

Theorem 17. For a function $f:(Z, \tau) \rightarrow X \times Y$, the following statements are equivalent:

(1) $f$ is binary pre- $\omega$-continuous.

(2) $f-1(A, B)$ is a closed set in $(Z, \tau)$ for each binary pre- $\omega$-closed set $(A, B)$ in $X \times Y$.

(3) For each $(x, y) \in(X, Y)$ and each binary pre- $\omega$-open set $(V, U)$ in $X \times Y$ containing $(f(x)$, $f(y))$ there exists an open set $(N, M)$ in $(Z, \tau)$ containing $(x, y)$ such that $f(N, M) \subset(V, U)$.

Proof. (1) $\Rightarrow(2)$ Let $(A, B)$ be any binary pre- $\omega$-closed set in $X \times Y$, then $(V, U)=(X, Y) \backslash(A, B)$ is a binary pre- $\omega$-open set in $X \times Y$ and since $f$ is binary pre- $\omega$-continuous, $f^{-1}((V, U))$ is an open subset in $(Z, \tau)$, but $f^{-1}((V, U))=f^{-1}((X$ $, Y) \backslash(A, B))=f^{-1}((X, Y)) \backslash f^{-1}((A, B))=(X, Y) \backslash f^{-1}((A, B))$ and hence, $f^{-1}((A, B))$ is a closed set in $(Z, \tau)$.

$(2) \Rightarrow(1)$ Let $(V, U)$ be any binary pre- $\omega$-open set in $X \times Y$, then $(A, B)=(X, Y) \backslash(V, U)$ is a binary pre- $\omega$-closed set in $X \times Y$. By hypothesis, we have $f^{-1}((A, B))$ is a closed set in $(Z, \tau)$, but $f^{-1}((A, B))=f^{-1}((X, Y) \backslash(V, U))=f^{-1}$ $((X, Y)) \backslash f^{-1}((V, U))=(X, Y) \backslash f^{-1}((V, U))$ and so, $f^{-1}((V, U))$ is an open set in $(Z, \tau)$. This shows that $f$ is binary pre- $\omega$-continuous.

$(1) \Rightarrow(3)$ Let $(x, y) \in(X, Y)$ and $(V, U)$ any binary pre- $\omega$-open set in $X \times Y$ such that $(f(x), f(y)) \in(V, U)$, then $(x, y)$ $\in f^{-1}((V, U))$ and since $f$ is a binary pre- $\omega$-continuous function, $f^{-1}((V, U))$ is an open set in $(Z, \tau)$. If $(N, M)=f^{-1}$ $((V, U))$, then $(N, M)$ is an open set in $(Z, \tau)$ containing $(x, y)$ such that $f((N, M))=f\left(f^{-1}((V, U))\right) \subset(V, U)$.

(3) $\Rightarrow$ (1) Let $(V, U)$ be any binary pre- $\omega$-open set in $X \times Y$ and $(x, y) \in f^{-1}((V, U))$, then $(f(x), f(y)) \in(V, U)$ and by (3) there exists an open set $(N, M) x$ in $(Z, \tau)$ such that $(x, y) \in(N, M)(x, y)$ and $f((N, M)(x, y)) \subset(V, U)$. Thus, $(x, y) \in$ $\left.(N, M)(x, y) \subset f^{-1}(f(N, M)(x, y))\right) \subset f^{-1}((V, U))$ and hence $f^{-1}((V, U))=\left\{(N, M)(x, y):(x, y) \in f^{-1}((V, U))\right\}$. Then, we have $f^{-1}((V, U))$ is an open set in $(Z, \tau)$ and so $f$ is a binary pre- $\omega$-continuous function. 
Proposition 3. Let $f:(Z, \tau) \rightarrow X \times Y$ be a binary pre- $\omega$-continuous if and only if for each $A \subseteq X$ and $B \subseteq Y, f^{-1}$ (Intp $\omega$ $(A, B)) \subseteq \operatorname{Intp} \omega\left(f^{-1}(A, B)\right)$.

Proof. Necessary: Let $f:(Z, \tau) \rightarrow X \times Y$ be a binary pre- $\omega$-continuous and let $A \subseteq X$ and $B \subseteq Y$. Then, Intp $\omega$ ( $A, B$ ) is a binary pre- $\omega$-open set of $(X, Y, M)$ and contained in $(A, B)$. Hence, $f^{-1}(\operatorname{Intp} \omega(A, B))$ is a pen set of $(Z, \tau)$. Now, Intp $\omega(A, B) \subseteq(A, B)$

$\Rightarrow f^{-1}(\operatorname{Intp} \omega(A, B)) \subseteq f^{-1}(A, B)$

$\Rightarrow \operatorname{Intp} \omega\left(f^{-1}(\operatorname{Intp} \omega(A, B))\right) \subseteq \operatorname{Intp} \omega\left(f^{-1}(A, B)\right)$

$\Rightarrow f^{-1}(\operatorname{Intp} \omega(A, B)) \subseteq \operatorname{Intp} \omega\left(f^{-1}(A, B)\right)$.

Sufficiency: Suppose that $f^{-1}(\operatorname{Intp} \omega(A, B)) \subseteq \operatorname{Intp} \omega\left(f^{-1}(A, B)\right)$ for each $A \subseteq X$ and $B \subseteq Y$. Now, let $(A, B) \in(X, Y$, $M)$, this implies that $\operatorname{Intp} \omega(A, B)=(A, B)$. Hence, $f^{-1}(A, B)=f^{-1}(\operatorname{Intp} \omega(A, B)) \subseteq \operatorname{Intp} \omega\left(f^{-1}(A, B)\right)$. Therefore, $\operatorname{Intp} \omega\left(f^{-1}(A, B)\right)$ is an open set of $(Z, \tau)$.

Definition 19. Let $f:(Z, \tau) \rightarrow X \times Y$ be a function, then $f$ is said to be:

(1) Strongly binary pre- $\omega$-continuous if the inverse image of every binary pre- $\omega$-closed set in $X \times Y$ is closed set in (Z, $\tau)$.

(2) Perfectly binary pre- $\omega$-continuous if the inverse image of every binary pre- $\omega$-closed set in $X \times Y$ is both open a closedin $(Z, \tau)$.

Theorem 18. Let $f:(Z, \tau) \rightarrow X \times Y$ be strongly binary pre- $\omega$-continuous, then $f$ is binary continuous.

Proof. Let $(V, U)$ be any binary closed set in $X \times Y$, since every binary closed set is binary pre-closed and it is well known that every binary pre-closed is binary pre- $\omega$-closed, $(V, U)$ is binary pre- $\omega$-closed set in $X \times Y$. Since $f$ is strongly binary pre- $\omega$-continuous, $f^{-1}(V, U)$ is closed set in $(Z, \tau)$. Therefore, $f$ is binary continuous.

Theorem 19. Let $f:(Z, \tau) \rightarrow X \times Y$ be perfectly binary pre- $\omega$-continuous, then $f$ is strongly binary pre- $\omega$-continuous. Proof.Let $(V, U)$ be any binary pre- $\omega$-closed set in $X \times Y$. Since $f$ is perfectly binary pre- $\omega$-continuous, $f^{-1}(V, U)$ is closed in $(Z, \tau)$. Therefore $f$ is strongly binary pre- $\omega$-continuous.

\section{Competing interests}

The authors declare that they have no competing interests.

\section{Authors' contributions}

All authors have contributed to all parts of the article. All authors read and approved the final manuscript.

\section{References}

[1] T. Chacko and D. Susha, Binary linear topological spaces, International Journal of mathematics and its Applications, 6(2A)(2018), 173-179.

[2] M. Ganster and I. Reily, A decomposition of continuity, Acta Math. Hungar., 56(1990), 229-301.

[3] H. Hdeib, $\omega$-closed mappings, Revista Colombiana de Mat., 16(1982), 65-78.

[4] S. Nithyanantha and P. Thangavelu, Topology between two sets,Journal of Mathematical Sciences and Computer Applications, 1(3), (2011), 95-107.

[5] A. Mehmood, M. Rahim, M. Zamir,F. Nadeem, S. Abduallah and S. Jabeen, Applications of soft semi-open sets to soft binary topology, Punjab University Journal of Mathematics, 51(??)(2019), 15-24.

[6] J. Mustafa, On binary generalized topological spaces, General Letters in mathematics,(2)3, (2017), 11-116. 